\title{
THEORETICAL STUDY OF THE $[2+2+2+1]$ CYCLOADDITION MECHANISM OF ENEDIYNES AND CARBON MONOXIDE CATALYZED BY RHODIUM
}

\section{Supporting Information}

M. Merced Montero-Campillo ${ }^{(a)}$, Jesús Rodríguez-Otero ${ }^{\left(a,{ }^{*}\right)}$,Enrique Cabaleiro-Lago ${ }^{(b)}$

(a) Departamento de Química Física, Facultade de Química, Universidade de Santiago de Compostela, Avda. das Ciencias s/n, 15782 Santiago de Compostela, Galicia (Spain).

(b) Departamento de Química Física, Facultade de Ciencias, Universidade de Santiago de Compostela, Campus de Lugo. Avda. Alfonso X El Sabio s/n 27002 Lugo, Galicia (Spain)

\section{Contents}

a) Table 1. Electronic energies and free energies for the catalyzed reaction in gas phase with B3LYP/6-31G(d,p)+LANL2DZ in atomic units.

b) Table 2. Electronic and free energies for the reaction without catalyst in gas phase at the B3LYP/6-31G(d,p) level in atomic units.

c) Table 3. Single point calculations of electronic energies with PCM method in dichloroethane solution at the B3LYP/6-31G $(\mathrm{d}, \mathrm{p})$ + LANL2DZ level in atomic units.

d) XYZ coordinates for molecules I1-I11, TS_Elim and Prod_Elim in gas phase for the rhodium-catalyzed cycloaddition in gas phase at B3LYP/6-31G(d,p) + LANL2DZ level 
Table 1. Electronic energies and free energies for the catalyzed reaction in gas phase with B3LYP/6-31G(d,p)+LANL2DZ in atomic units.

\begin{tabular}{|c|c|c|c|c|}
\hline & $\boldsymbol{E}_{\text {elec }}(\boldsymbol{a} . \boldsymbol{u})$ & $\boldsymbol{E}_{\text {elec }}+\boldsymbol{Z P E}(\boldsymbol{a . u .})$ & $\Delta \boldsymbol{G}(\boldsymbol{a . u})$ & $\Delta \boldsymbol{G}+\boldsymbol{Z P E}$ (a.u) \\
\hline $\mathbf{I 1}+\mathbf{C O}$ & -1374.30342 & -1374.06445 & -1374.13563 & -1373.89667 \\
\hline $\mathbf{T S 2}+\mathbf{C O}$ & -1374.26763 & -1374.03011 & -1374.10038 & -1373.86287 \\
\hline $\mathbf{I 3}+\mathbf{C O}$ & -1374.35018 & -1374.10897 & -1374.17847 & -1373.93726 \\
\hline $\mathbf{I 4}+\mathbf{C O}$ & -1374.34993 & -1374.10799 & -1374.17438 & -1373.93246 \\
\hline $\mathbf{I 5}$ & -1374.37117 & -1374.12629 & -1374.17662 & -1373.93173 \\
\hline TS6 & -1374.31759 & -1374.07185 & -1374.12060 & -1373.87666 \\
\hline $\mathbf{I 7}$ & -1374.39324 & -1374.14632 & -1374.19655 & -1373.94963 \\
\hline TS8 & -1374.37637 & -1374.12949 & -1374.17933 & -1373.93246 \\
\hline $\mathbf{I 9}$ & -1374.40412 & 1374.15186 & -1374.20124 & -1373.95168 \\
\hline TS10 & -1374.34942 & -1374.10108 & -1374.15029 & -1373.90195 \\
\hline $\mathbf{I 1 1}$ & -1374.41356 & -1374.16237 & -1374.21367 & -1373.96248 \\
\hline
\end{tabular}


Table 2. Electronic and free energies for the reaction without catalyst in gas phase at the B3LYP/6-31G(d,p) level in atomic units.

\begin{tabular}{|c|c|c|c|c|}
\hline & $E_{\text {elec }}($ a.u. $)$ & $E_{\text {elec }}+Z P E$ (a.u.) & $\Delta G$ (u.a.) & $\Delta G+Z P E$ (u.a.) \\
\hline I1 & -691.18273 & -690.95454 & -691.011683 & -690.783493 \\
\hline TS2 & -691.13753 & -690.90790 & -690.954460 & -690.724827 \\
\hline I3 & -691.29497 & -691.05881 & -691.103227 & -690.867075 \\
\hline I4 & -691.29193 & -691.05564 & -691.099513 & -690.863225 \\
\hline TS5_1 & -691.21716 & -690.98226 & -691.029684 & -690.793552 \\
\hline TS5_2 & -691.22685 & -690.99072 & -691.023333 & -690.788431 \\
\hline P1_a & -691.26300 & -691.02380 & -691.063107 & -690.823905 \\
\hline P1_b & -691.33745 & -691.09688 & -691.137250 & -690.896680 \\
\hline P2 & -691.30175 & -691.06000 & -691.099362 & -690.857618 \\
\hline
\end{tabular}


Table 3. Single point calculations of electronic energies with PCM method in dichloroethane solution at the B3LYP/6-31G(d,p) + LANL2DZ level in atomic units.

\begin{tabular}{|c|c|c|}
\hline & $\boldsymbol{E}_{\text {elec }}(\boldsymbol{a . u .})$ & $\boldsymbol{E}_{\text {elec }}+\boldsymbol{Z P E}$ (a.u.) \\
\hline $\mathbf{I 1}+\mathbf{C O}$ & -1374.29720 & -1374.05918 \\
\hline TS2 + CO & -1374.26404 & -1374.02719 \\
\hline $\mathbf{I 3}+\mathbf{C O}$ & -1374.34677 & -1374.10620 \\
\hline $\mathbf{I 4 + C O}$ & -1374.34624 & -1374.10491 \\
\hline $\mathbf{I 5}$ & -1374.36947 & -1374.12517 \\
\hline TS6 & -1374.31471 & -1374.07128 \\
\hline $\mathbf{I 7}$ & -1374.39144 & -1374.14493 \\
\hline TS8 & -1374.37701 & -1374.13087 \\
\hline $\mathbf{I 9}$ & -1374.40280 & -1374.15391 \\
\hline TS10 & -1373.35099 & -1374.10353 \\
\hline I11 & -1374.42629 & -1374.17595 \\
\hline
\end{tabular}


XYZ coordinates for molecules I1-I11, TS_Elim and Prod_Elim in gas phase for the rhodium-catalyzed cycloaddition in gas phase at B3LYP/6-31G(d,p) + LANL2DZ level.

\begin{tabular}{|c|c|c|c|}
\hline \multicolumn{4}{|l|}{ I1 } \\
\hline $\mathrm{C}$ & 0.000130 & 0.000505 & -0.001906 \\
\hline $\mathrm{C}$ & -0.001074 & -0.005208 & 2.801087 \\
\hline $\mathrm{C}$ & 1.475676 & -0.000015 & 0.145731 \\
\hline $\mathrm{C}$ & -1.163774 & -0.388418 & -0.243146 \\
\hline $\mathrm{C}$ & -1.146036 & -0.262561 & 3.150196 \\
\hline $\mathrm{C}$ & 1.445407 & 0.139041 & 2.533014 \\
\hline 0 & 1.884667 & -0.574215 & 1.382035 \\
\hline $\mathrm{Rh}$ & -1.305957 & 1.229990 & 1.201650 \\
\hline $\mathrm{C}$ & -2.270018 & -1.171858 & -0.850233 \\
\hline $\mathrm{H}$ & 1.867136 & 1.023069 & 0.045167 \\
\hline $\mathrm{H}$ & 1.930136 & -0.623183 & -0.631629 \\
\hline $\mathrm{C}$ & -2.445328 & -0.625115 & 3.714005 \\
\hline $\mathrm{H}$ & 1.706463 & 1.204541 & 2.456474 \\
\hline $\mathrm{H}$ & 2.001985 & -0.288141 & 3.373579 \\
\hline $\mathrm{Cl}$ & -2.146822 & 2.803629 & 2.781408 \\
\hline $\mathrm{C}$ & -1.743327 & 2.444873 & -0.136583 \\
\hline $\mathrm{H}$ & -3.008813 & -0.494879 & -1.288451 \\
\hline $\mathrm{H}$ & -1.852492 & -1.793860 & -1.660188 \\
\hline 0 & -2.973722 & -1.969106 & 0.081727 \\
\hline $\mathrm{H}$ & -2.301565 & -1.232009 & 4.614885 \\
\hline $\mathrm{H}$ & -2.996034 & 0.282920 & 3.975341 \\
\hline $\mathrm{H}$ & -3.035231 & -1.203460 & 2.996666 \\
\hline 0 & -1.992958 & 3.209251 & -0.957645 \\
\hline $\mathrm{C}$ & -2.227613 & -3.073643 & 0.570229 \\
\hline $\mathrm{H}$ & -1.334480 & -2.726894 & 1.115409 \\
\hline $\mathrm{H}$ & -1.861200 & -3.685361 & -0.274635 \\
\hline $\mathrm{C}$ & -3.078644 & -3.918596 & 1.471859 \\
\hline $\mathrm{C}$ & -4.369133 & -3.710917 & 1.730648 \\
\hline $\mathrm{H}$ & -2.553929 & -4.759301 & 1.924611 \\
\hline $\mathrm{H}$ & -4.921507 & -4.368016 & 2.394979 \\
\hline $\mathrm{H}$ & -4.907182 & -2.883006 & 1.281600 \\
\hline \multicolumn{4}{|c|}{ TS2 } \\
\hline $\mathrm{C}$ & -0.000035 & -0.000214 & -0.001376 \\
\hline $\mathrm{C}$ & -0.000772 & -0.002425 & 2.103716 \\
\hline $\mathrm{C}$ & 1.494655 & 0.001834 & -0.059495 \\
\hline $\mathrm{C}$ & -1.159959 & -0.093485 & -0.525141 \\
\hline $\mathrm{C}$ & -1.163470 & -0.189053 & 2.601483 \\
\hline $\mathrm{C}$ & 1.474347 & -0.066256 & 2.255582 \\
\hline 0 & 2.068449 & -0.604221 & 1.077225 \\
\hline $\mathrm{Rh}$ & -1.604962 & 1.146544 & 1.112165 \\
\hline $\mathrm{C}$ & -1.922658 & -0.530150 & -1.733965 \\
\hline $\mathrm{H}$ & 1.848414 & 1.040040 & -0.176687 \\
\hline $\mathrm{H}$ & 1.812690 & -0.575008 & -0.934267 \\
\hline $\mathrm{C}$ & -2.068245 & -0.731372 & 3.626114 \\
\hline $\mathrm{H}$ & 1.862738 & 0.943911 & 2.463541 \\
\hline $\mathrm{H}$ & 1.754028 & -0.727553 & 3.080472 \\
\hline $\mathrm{Cl}$ & -1.728665 & 2.843466 & 2.844658 \\
\hline $\mathrm{C}$ & -3.499581 & 1.441292 & 0.748707 \\
\hline $\mathrm{H}$ & -1.990930 & 0.309946 & -2.433786 \\
\hline
\end{tabular}




\begin{tabular}{|c|c|c|c|}
\hline $\mathrm{H}$ & -1.371836 & -1.343977 & -2.234000 \\
\hline 0 & -3.252553 & -0.920798 & -1.469565 \\
\hline $\mathrm{H}$ & -1.529199 & -1.428304 & 4.278793 \\
\hline $\mathrm{H}$ & -2.477993 & 0.088340 & 4.224362 \\
\hline $\mathrm{H}$ & -2.905141 & -1.265704 & 3.163667 \\
\hline 0 & -4.629635 & 1.556066 & 0.608164 \\
\hline $\mathrm{C}$ & -3.347179 & -2.144474 & -0.759374 \\
\hline $\mathrm{H}$ & -2.781120 & -2.081353 & 0.186023 \\
\hline $\mathrm{H}$ & -2.881923 & -2.957706 & -1.346340 \\
\hline $\mathrm{C}$ & -4.779570 & -2.481759 & -0.468968 \\
\hline $\mathrm{C}$ & -5.829960 & -1.730470 & -0.794136 \\
\hline $\mathrm{H}$ & -4.919072 & -3.428153 & 0.052820 \\
\hline $\mathrm{H}$ & -6.840174 & -2.040806 & -0.546352 \\
\hline $\mathrm{H}$ & -5.709288 & -0.787160 & -1.315127 \\
\hline \multicolumn{4}{|l|}{ I3 } \\
\hline C & -0.000651 & -0.000798 & -0.002232 \\
\hline $\mathrm{C}$ & -0.000453 & -0.001081 & 1.456932 \\
\hline $\mathrm{C}$ & 1.451810 & 0.001004 & -0.438081 \\
\hline $\mathrm{C}$ & -1.180405 & 0.036123 & -0.631381 \\
\hline $\mathrm{C}$ & -1.177811 & 0.036568 & 2.091753 \\
\hline $\mathrm{C}$ & 1.451429 & -0.008927 & 1.894883 \\
\hline 0 & 2.167474 & -0.421801 & 0.725625 \\
\hline $\mathrm{Rh}$ & -2.691731 & 0.144043 & 0.732362 \\
\hline $\mathrm{C}$ & -1.555220 & 0.168435 & -2.070583 \\
\hline $\mathrm{H}$ & 1.767647 & 1.010718 & -0.748687 \\
\hline $\mathrm{H}$ & 1.680363 & -0.697346 & -1.248887 \\
\hline $\mathrm{C}$ & -1.523094 & 0.125850 & 3.536127 \\
\hline $\mathrm{H}$ & 1.774269 & 0.994907 & 2.217029 \\
\hline $\mathrm{H}$ & 1.678279 & -0.719044 & 2.695724 \\
\hline $\mathrm{Cl}$ & -2.896664 & 2.453836 & 0.755520 \\
\hline $\mathrm{C}$ & -2.792215 & -1.741198 & 0.726020 \\
\hline $\mathrm{H}$ & -2.039851 & 1.136492 & -2.239326 \\
\hline $\mathrm{H}$ & -0.636136 & 0.139010 & -2.683745 \\
\hline 0 & -2.490270 & -0.806641 & -2.514342 \\
\hline $\mathrm{H}$ & -0.613737 & 0.060699 & 4.147723 \\
\hline $\mathrm{H}$ & -2.016227 & 1.079274 & 3.760326 \\
\hline $\mathrm{H}$ & -2.199816 & -0.678213 & 3.850086 \\
\hline 0 & -2.853274 & -2.887387 & 0.734720 \\
\hline $\mathrm{C}$ & -1.928252 & -2.094024 & -2.704268 \\
\hline $\mathrm{H}$ & -1.420706 & -2.439292 & -1.787922 \\
\hline $\mathrm{H}$ & -1.150818 & -2.051744 & -3.489452 \\
\hline $\mathrm{C}$ & -2.988428 & -3.081458 & -3.094391 \\
\hline $\mathrm{C}$ & -4.276285 & -2.795652 & -3.277794 \\
\hline $\mathrm{H}$ & -2.625238 & -4.099758 & -3.229398 \\
\hline $\mathrm{H}$ & -4.987491 & -3.565289 & -3.560841 \\
\hline $\mathrm{H}$ & -4.651968 & -1.785488 & -3.155560 \\
\hline \multicolumn{4}{|c|}{ I 4} \\
\hline $\mathrm{C}$ & 0.000683 & -0.000181 & 0.00001 \\
\hline $\mathrm{C}$ & 0.000963 & -0.000761 & 1.455571 \\
\hline $\mathrm{C}$ & 1.449972 & 0.000438 & -0.439895 \\
\hline $\mathrm{C}$ & -1.171681 & 0.108946 & -0.642027 \\
\hline $\mathrm{C}$ & -1.180426 & 0.114163 & 2.082111 \\
\hline $\mathrm{C}$ & 1.452111 & -0.024010 & 1.893424 \\
\hline 0 & 2.172294 & -0.422401 & 0.719002 \\
\hline $\mathrm{Rh}$ & -2.712948 & 0.138061 & 0.708728 \\
\hline $\mathrm{C}$ & -1.360194 & 0.509659 & -2.081260 \\
\hline
\end{tabular}




\begin{tabular}{|c|c|c|c|}
\hline $\mathrm{H}$ & 1.767551 & 1.009586 & -0.754948 \\
\hline $\mathrm{H}$ & 1.673562 & -0.697066 & -1.254327 \\
\hline $\mathrm{C}$ & -1.475045 & 0.290266 & 3.532503 \\
\hline $\mathrm{H}$ & 1.785463 & 0.970302 & 2.234697 \\
\hline $\mathrm{H}$ & 1.673397 & -0.749513 & 2.682534 \\
\hline $\mathrm{Cl}$ & -2.740377 & 2.495997 & 0.898626 \\
\hline $\mathrm{C}$ & -2.625733 & -1.746805 & 0.818565 \\
\hline $\mathrm{H}$ & -1.151458 & 1.580265 & -2.190201 \\
\hline $\mathrm{H}$ & -0.598719 & -0.040228 & -2.665921 \\
\hline 0 & -2.638382 & 0.346890 & -2.682859 \\
\hline $\mathrm{H}$ & -0.553884 & 0.258334 & 4.128582 \\
\hline $\mathrm{H}$ & -1.958269 & 1.259760 & 3.704173 \\
\hline $\mathrm{H}$ & -2.151426 & -0.483711 & 3.917530 \\
\hline 0 & -2.535590 & -2.888012 & 0.914178 \\
\hline $\mathrm{C}$ & -3.335790 & -0.844599 & -2.399585 \\
\hline $\mathrm{H}$ & -2.654325 & -1.673084 & -2.156312 \\
\hline $\mathrm{H}$ & -3.870547 & -1.136227 & -3.315082 \\
\hline $\mathrm{C}$ & -4.348214 & -0.649557 & -1.294619 \\
\hline $\mathrm{C}$ & -4.737404 & 0.560548 & -0.838683 \\
\hline $\mathrm{H}$ & -4.838867 & -1.553055 & -0.932316 \\
\hline $\mathrm{H}$ & -5.532430 & 0.657591 & -0.105932 \\
\hline $\mathrm{H}$ & -4.327578 & 1.471298 & -1.25687 \\
\hline \multicolumn{4}{|l|}{ I5 } \\
\hline 0 & -0.000732 & -0.000726 & -0.000893 \\
\hline C & -0.000126 & 0.001504 & 1.430688 \\
\hline $\mathrm{C}$ & 1.468214 & 0.001101 & 1.808821 \\
\hline $\mathrm{C}$ & 2.213054 & -0.377610 & 0.619152 \\
\hline $\mathrm{C}$ & 1.193032 & -0.642882 & -0.466413 \\
\hline C & 3.544383 & -0.534442 & 0.721414 \\
\hline $\mathrm{C}$ & 4.362076 & -1.214141 & -0.344572 \\
\hline 0 & 5.783829 & -1.115181 & -0.273730 \\
\hline $\mathrm{C}$ & 6.308537 & 0.156198 & 0.014978 \\
\hline $\mathrm{C}$ & 6.664165 & 0.299216 & 1.476033 \\
\hline $\mathrm{C}$ & 6.735482 & -0.741772 & 2.332901 \\
\hline $\mathrm{C}$ & 2.127266 & 0.175404 & 2.961019 \\
\hline $\mathrm{Rh}$ & 4.181958 & -0.079414 & 2.651251 \\
\hline C & 4.124776 & 1.751444 & 2.140666 \\
\hline 0 & 4.045447 & 2.848507 & 1.818459 \\
\hline C & 1.586276 & 0.410390 & 4.330117 \\
\hline $\mathrm{Cl}$ & 3.921472 & -2.437177 & 3.148335 \\
\hline $\mathrm{H}$ & 0.498215 & 0.546822 & 4.289435 \\
\hline $\mathrm{H}$ & 1.788399 & -0.448135 & 4.982650 \\
\hline $\mathrm{H}$ & 2.015932 & 1.299218 & 4.809780 \\
\hline $\mathrm{H}$ & -0.510179 & -0.894099 & 1.823826 \\
\hline $\mathrm{H}$ & -0.551927 & 0.885495 & 1.766425 \\
\hline $\mathrm{H}$ & 4.172331 & -2.293245 & -0.308184 \\
\hline $\mathrm{H}$ & 4.021169 & -0.851269 & -1.33174 \\
\hline $\mathrm{H}$ & 5.632303 & 0.965280 & -0.299110 \\
\hline $\mathrm{H}$ & 7.231393 & 0.276768 & -0.572222 \\
\hline $\mathrm{H}$ & 7.119056 & -0.613922 & 3.339372 \\
\hline $\mathrm{H}$ & 6.504188 & -1.747877 & 2.004944 \\
\hline $\mathrm{H}$ & 6.976144 & 1.292954 & 1.798761 \\
\hline $\mathrm{H}$ & 1.023569 & -1.724677 & -0.606238 \\
\hline $\mathrm{H}$ & 1.450386 & -0.212109 & -1.44049 \\
\hline $\mathrm{C}$ & 4.693516 & 0.098450 & 4.63960 \\
\hline 0 & 4.963831 & 0.074097 & 5.74696 \\
\hline
\end{tabular}


TS 6

0

$$
0.000804
$$

0.002727

$-0.001269$

C $\quad 0.000789$

0.001591

1.469781

$-0.000887$

1.430153

C $\quad 2.235643$

$-0.234244$

1.815118

$-0.553756$

0.629183

1.229401

$-0.388992$

$-0.465073$

$-1.465479$

$-1.181069$

0.658612

$-0.287293$

$-0.790620$

0.210394

$-0.909784$

5.541438

0.815516

0.447530

6.051336

0.494688

$-0.078382$

1.574886

3.031129

$-0.078537$

2.742035

1.779780

2.823647

2. 897351

2.966896

0.046237

4.343932

$-2.483457$

0.186957

2. 827670

$-0.848559$

4. 246126

4.952106

0.891602

4.922337

1.785140

$-0.886314$

1.821222

$-0.522234$

0.891092

1. 767267

$-2.429334$

0.223469

4. 203491

$-1.553471$

$-1.136819$

4.855117

0.611253

$-1.674761$

6.570737

0.456301

$-1.194326$

1.317699

2.149168

6.678718

$-0.387592$

1.488566

1.820491

0.416428

1.139098

$-1.644061$

$-0.613657$

1. 468583

$-0.099833$

$-1.432629$

4. 947253

$-0.192429$

4.582058

$-0.374459$

5.636315

I 7

C $\quad-0.001608$

0.000040

1.019553

0.806231

0.595530

$-0.026130$

1. 682717

$-1.458856$

$-1.569212$

$-1.854636$

$-1.346637$

$-2.218481$

0.921494

$\mathrm{Rh} \quad 3.035531$

Cl 2.640323

C $\quad 3.201213$

o 3.275780

C $\quad 0.829773$

$\mathrm{H} \quad 0.661332$

$\mathrm{H} \quad 0.017619$

O $\quad 2.095087$

C $\quad 2.593425$
0.003757

0.001583

0.000153

$-0.126053$

0.846006

$-0.803318$

$-0.540692$

$-0.216855$

$-1.174137$

0.577936

$-0.570710$

$-0.226834$

0.458794

$-0.210341$

$-2.514752$

1. 612459

2.719579

0.370111

$-0.645422$

1. 012085

0.807457

1. 748858
$-0.000857$

1. 465307

2.335224

3. 829477

4. 295648

4.057233

4. 349351

1.877160

2. 415288

2.520414

$-0.401166$

0.673349

$-0.857453$

2.089391

1.575115

2.582399

2.873980

$-2.370771$

$-2.744276$

$-2.759348$

$-2.858618$

$-1.925146$ 


$\begin{array}{rr}\mathrm{H} & 2.134324 \\ \mathrm{H} & 3.672494 \\ \mathrm{C} & 2.191539 \\ \mathrm{C} & 3.305293 \\ \mathrm{H} & 4.256498 \\ \mathrm{H} & 3.412884 \\ \mathrm{H} & 1.976661 \\ \mathrm{H} & -1.289523 \\ \mathrm{H} & -1.757911 \\ \mathrm{C} & 5.035024 \\ \mathrm{O} & 6.072295\end{array}$

2.740377
1.839998
1.210195
0.362652
0.900450
-0.605976
2.053336
-1.666043
-0.139539
-0.835179
-1.293443

$$
\begin{array}{r}
-2.075242 \\
-2.078552 \\
-0.516480 \\
0.071694 \\
0.089341 \\
-0.416740 \\
0.150593 \\
-0.529723 \\
-1.319897 \\
2.179879 \\
2.282661
\end{array}
$$

TS 8

C $\quad-0.001364$

C $\quad-0.000588$

C $\quad 0.989086$

0.003425

$-0.000473$

$-0.000821$

1. 462102

$-0.001157$

2.390302

0.664782

$-0.178676$

3. 861596

0.625739

4. 232594

$-1.135652$

4. 029191

0.157442

$-0.178317$

4.488421

$-1.456615$

$-0.208553$

1.869125

$-1.581781$

$-1.220561$

0.513388

2.293096

$-1.798060$

$-0.383539$

$-1.414934$

$-0.041748$

2. 619414

$-2.234631$

0.262188

$-0.413712$

0.695911

$-0.890864$

0.971757

$-0.760417$

1.973667

2. 401919

$-3.120461$

1. 052477

2. 192287

.940884

2. 326882

2.575310

0.122628

$-2.392433$

$-2.695321$

$-2.780923$

0.922074

$-2.947111$

0.223039

1. 101687

$-2.108420$

2.154399

$-2.327321$

0.944856

$-2.294324$

0.764250

$-0.649777$

$-0.249400$

0.133337

$-0.030010$

$-0.075795$

$-0.492849$

3. 271524

2. 373170

$-1.236468$

1.682602

$-0.054858$

$-1.463730$

$-0.635872$

$-1.476759$

0.165597

$-1.238438$

$-1.287053$

2.102360

2. 258010

I9

C $\quad 0.000055$

C $\quad-0.000267$

$-1.522128$

$-0.001274$

$-0.000384$

$-0.000838$

$-0.000022$

1. 466945

2. 400088

0.980933

0.021716

3.893025

1.144475

0.914679

4.344943

$-0.367035$

0.012353

4.110930

$-0.847968$

4.388110

1.148293

$-0.084831$

1.870292

$-1.064107$

2. 341088

$-1.667157$

0.697757

2. 572247 


$\begin{array}{rr}\mathrm{C} & -1.421111 \\ \mathrm{O} & -2.232772 \\ \mathrm{C} & 0.941924 \\ \mathrm{Rh} & 3.205019 \\ \mathrm{Cl} & 1.464671 \\ \mathrm{C} & 2.452290 \\ \mathrm{O} & 3.190433 \\ \mathrm{C} & 0.669067 \\ \mathrm{H} & 0.437462 \\ \mathrm{H} & -0.180505 \\ \mathrm{O} & 1.861049 \\ \mathrm{C} & 2.576522 \\ \mathrm{H} & 2.172221 \\ \mathrm{H} & 3.619506 \\ \mathrm{C} & 2.372513 \\ \mathrm{C} & 3.385587 \\ \mathrm{H} & 4.401645 \\ \mathrm{H} & 3.228947 \\ \mathrm{H} & 2.496041 \\ \mathrm{H} & -1.506566 \\ \mathrm{H} & -1.772252 \\ \mathrm{C} & 4.946890 \\ \mathrm{O} & 6.029099\end{array}$

$$
\begin{array}{r}
-0.369175 \\
0.069013 \\
0.284915 \\
-1.525161 \\
-2.980452 \\
0.029641 \\
0.815750 \\
0.238908 \\
-0.769304 \\
0.893496 \\
0.678481 \\
1.465713 \\
2.489869 \\
1.518093 \\
0.745524 \\
-0.400644 \\
-0.007865 \\
-1.162407 \\
1.448409 \\
-1.459591 \\
0.130380 \\
-0.957063 \\
-0.686661
\end{array}
$$

TS10

C

C

C

$\mathrm{H}$

$\mathrm{H}$

$\mathrm{H}$

C

$\mathrm{H}$

$\mathrm{H}$

C

O

C

$\mathrm{Rh}$

Cl

C

$\mathrm{O}$

C

$\mathrm{H}$

$\mathrm{H}$

O

C

$\mathrm{H}$

$\mathrm{H}$

C

C

$\mathrm{H}$

$\mathrm{H}$

$\mathrm{H}$

$\mathrm{H}$

$\mathrm{H}$

C

O

$$
\begin{array}{rr}
0.002424 & -0.004405 \\
0.001171 & 0.000868 \\
1.026002 & 0.004206 \\
0.777046 & 0.063591 \\
1.317059 & 0.907688 \\
-0.283298 & 0.166768 \\
1.146337 & -0.840544 \\
-1.466604 & -0.100665 \\
-1.645018 & -1.068065 \\
-1.795492 & 0.697239 \\
-1.389487 & -0.421561 \\
-2.220236 & 0.002136 \\
0.995839 & 0.402304 \\
2.868591 & -2.177622 \\
0.879324 & -3.185275 \\
2.491048 & -0.109756 \\
3.318187 & 0.335571 \\
1.024605 & 0.400834 \\
0.708577 & -0.548439 \\
0.383805 & 1.201980 \\
2.391902 & 0.616733 \\
2.945776 & 1.438736 \\
2.671006 & 2.495583 \\
4.034244 & 1.350811 \\
2.319422 & 0.940475 \\
3.198266 & -0.139786 \\
4.184310 & 0.236754 \\
3.302664 & -0.939193 \\
2.172819 & 1.770925 \\
-1.444869 & -1.512505 \\
-1.741019 & 0.072734 \\
4.614028 & -2.075564 \\
5.689263 & -2.087950 \\
& \\
\hline & \\
1.36
\end{array}
$$

$$
\begin{array}{r}
-0.396577 \\
0.685255 \\
-0.918117 \\
1.098229 \\
0.510517 \\
2.160301 \\
2.697019 \\
-2.414957 \\
-2.779080 \\
-2.676251 \\
-3.057230 \\
-2.117517 \\
-2.059889 \\
-2.441063 \\
-0.757527 \\
-0.638813 \\
-0.705657 \\
-1.406709 \\
0.071007 \\
-0.543495 \\
-1.304664 \\
1.583772 \\
1.853843
\end{array}
$$

0.000943

1. 463056

2. 352130

3. 847337

4.285797

4.078602

4. 344146

1.860287

2.358555

2.534863

$-0.421660$

0.659046

$-0.798713$

1.322519

0.626952

2.069719

2.839083

$-2.312226$

$-2.754997$

$-2.723151$

$-2.657396$

$-1.640733$

$-1.792912$

$-1.686307$

$-0.313119$

0.341014

0.600950

$-0.417244$

0.388354

$-0.564258$

$-1.332656$

1.996640

2.404940 
I11

C $\quad 0.000221$

0.001942

0.000415

$-0.001168$

$-0.000407$

1.459437

C $\quad 1.016821$

$-0.002521$

2.376535

$-0.028831$

3. 862770

0.727078

0.709673

4. 388207

$-0.320537$

0.178857

4. 081505

0.983761

$-1.003394$

4. 292210

$-1.466485$

$-0.117977$

1.857587

$-1.640894$

$-1.789789$

$-1.083511$

2.362271

$-1.400966$

0.676870

2.538850

$-0.387254$

$-2.229118$

$-0.016060$

$-0.432382$

0.382131

0.664645

$-0.805657$

$-0.948674$

2. 935010

$-3.021421$

1. 934423

$-0.146273$

2.032027

$-0.325232$

3. 012960

0.377633

$-2.315255$

$-0.482992$

$-2.765394$

1. 296101

$-2.712366$

0.315829

$-2.652694$

1. 055935

$-1.663611$

2.124326

$-1.927206$

0.678140

$-1.640470$

0.857397

$-0.327966$

$-0.139763$

0.633826

0.068702

0.750405

$-1.166476$

0.246095

1.820952

0.191139

2. 245078

$-1.470098$

$-1.470593$

$-0.632679$

$-1.752775$

0.147521

$-1.319610$

0.637385

3.732683

1. 617405

4. 223635

\section{TS_Elim}

$\begin{array}{rr}\text { C } & -2.079899 \\ \mathrm{C} & -1.173434 \\ \mathrm{C} & 0.051130 \\ \mathrm{C} & 0.660949 \\ \mathrm{H} & 0.970071 \\ \mathrm{H} & -0.063597 \\ \mathrm{H} & 1.548249 \\ \mathrm{C} & -1.843727 \\ \mathrm{H} & -1.167081 \\ \mathrm{H} & -2.142528 \\ \mathrm{C} & -2.927161 \\ \mathrm{O} & -3.018189 \\ \mathrm{C} & -2.206535 \\ \mathrm{Rh} & 1.451218 \\ \mathrm{Cl} & 0.247882 \\ \mathrm{C} & 2.662386 \\ \mathrm{O} & 3.453392 \\ \mathrm{C} & -3.047224 \\ \mathrm{H} & -2.961752 \\ \mathrm{H} & -4.115469 \\ \mathrm{O} & -2.523995\end{array}$

$$
\begin{array}{rr}
0.539257 & 0.326745 \\
1.510986 & -0.271670 \\
1.311704 & -0.804628 \\
2.289317 & -1.782600 \\
1.773563 & -2.700591 \\
3.054297 & -2.081360 \\
2.797916 & -1.387673 \\
2.868472 & -0.055168 \\
3.547563 & 0.486259 \\
3.361727 & -0.990301 \\
1.306140 & 1.309159 \\
2.611296 & 0.713340 \\
-0.696143 & -0.164732 \\
0.012662 & 0.105147 \\
-0.413379 & 2.189635 \\
0.204292 & -1.310247 \\
0.271381 & -2.143652 \\
-1.854214 & 0.315281 \\
-2.026177 & 1.392328 \\
-1.706312 & 0.070192 \\
-2.997054 & -0.356478
\end{array}
$$




$\begin{array}{rrrr}\mathrm{C} & -2.040633 & -2.569933 & -1.619342 \\ \mathrm{H} & -2.857169 & -2.522821 & -2.360056 \\ \mathrm{H} & -1.306662 & -3.305540 & -1.964421 \\ \mathrm{C} & -1.454823 & -1.140205 & -1.413756 \\ \mathrm{C} & 0.055006 & -1.137196 & -1.330634 \\ \mathrm{H} & 0.520788 & -0.983175 & -2.299029 \\ \mathrm{H} & 0.448122 & -2.022727 & -0.822870 \\ \mathrm{H} & -1.755145 & -0.483425 & -2.239154 \\ \mathrm{H} & -2.445581 & 1.355166 & 2.297440 \\ \mathrm{H} & -3.943526 & 0.922229 & 1.427144 \\ \mathrm{C} & 2.871224 & -0.668585 & 1.328912 \\ \mathrm{O} & 3.676170 & -1.026120 & 2.056085 \\ & & & \\ \mathrm{PrOd} \mathrm{E} 1 \mathrm{im} & & & -0.000191 \\ \mathrm{C} & -0.000239 & -0.001114 & -0.0693 \\ \mathrm{C} & 0.000237 & -0.001358 & 1.469043 \\ \mathrm{C} & 1.156631 & -0.000279 & 2.211384 \\ \mathrm{C} & 1.215895 & 0.384083 & 3.670563 \\ \mathrm{H} & 1.911550 & -0.251984 & 4.227146 \\ \mathrm{H} & 1.593700 & 1.412661 & 3.750594 \\ \mathrm{H} & 0.242494 & 0.350619 & 4.163647 \\ \mathrm{C} & -1.450562 & 0.257163 & 1.828308 \\ \mathrm{H} & -1.803862 & -0.171174 & 2.766709 \\ \mathrm{H} & -1.617147 & 1.349621 & 1.868097 \\ \mathrm{C} & -1.418525 & -0.256035 & -0.448602 \\ \mathrm{O} & -2.189747 & -0.334243 & 0.763028 \\ \mathrm{C} & 1.119629 & 0.294891 & -0.665923 \\ \mathrm{Rh} & 0.369754 & -2.457733 & 2.165434 \\ \mathrm{Cl} & 1.474427 & -3.018330 & 0.111997 \\ \mathrm{C} & -0.518712 & -2.198236 & 3.794152 \\ \mathrm{O} & -1.076110 & -2.085982 & 4.796537 \\ \mathrm{C} & 1.437825 & 0.314495 & -2.138458 \\ \mathrm{H} & 1.245120 & -0.675959 & -2.580895 \\ \mathrm{H} & 0.865152 & 1.057874 & -2.707817 \\ \mathrm{O} & 2.823333 & 0.657127 & -2.224282 \\ \mathrm{C} & 3.442354 & 0.288673 & -0.988396 \\ \mathrm{H} & 4.375827 & 0.851585 & -0.908864 \\ \mathrm{H} & 3.663556 & -0.789039 & -0.958461 \\ \mathrm{C} & 2.393950 & 0.649708 & 0.076213 \\ \mathrm{C} & 2.501676 & -0.007342 & 1.456965 \\ \mathrm{H} & 3.239532 & 0.522760 & 2.069061 \\ \mathrm{H} & 2.861121 & -1.035535 & 1.351657 \\ \mathrm{H} & 2.402932 & 1.747166 & 0.184778 \\ \mathrm{H} & -1.536315 & -1.205217 & -0.983035 \\ \mathrm{H} & -1.796995 & 0.554756 & -1.090209 \\ \mathrm{C} & 0.270095 & -4.327178 & 2.328092 \\ \mathrm{O} & 0.224474 & -5.467658 & 2.422796\end{array}$

